\title{
Simulation of Engine Power based on Mean Value Model
}

\author{
Hui Meng, Liqiang Wang, Zongqi Han, andShubin Lei, \\ ${ }^{1}$ College of Electrical Engineering, Yanshan University, Qinhuangdao, 066004, China \\ ${ }^{2}$ College of Vehicle and Energy Engineering, Yanshan University, Qinhuangdao,066004, China \\ ${ }^{3}$ College of Vehicle and Energy Engineering, Yanshan University, Qinhuangdao,066004, China; \\ ${ }^{4}$ College of Vehicle and Energy Engineering, Yanshan University, Qinhuangdao, 066004 ,China
}

\begin{abstract}
The intake system sub-model, the fuel dynamics sub-model and the power output sub-model has been studied in this paper based on Elbert Hendricks engine mean model. Engine output characteristics using computational model is established in MATLAB/Simulink. Parameter of the modeling and simulation process is obtained through experiments, and finally, the engine bench test is done, and compared it with the simulation curve in same conditions. The results show that the model calculation has smaller error, higher precision, and which can meet the basic requirements of the engine modeling in actual control.
\end{abstract}

Keywords: - Engine, modeling, simulation, bench test, Simulink.

\section{INTRODUCTION}

The first thing needs to do is evaluating key design parameters and predict performance when design and control a new engine. The thermodynamic model is commonly used internal combustion engine as a series of controlled volume (thermal system) with energy and mass exchange [1]. Huge amount of calculation limited its practical application. The analogy method is mainly used for the comparison of major performance parameters, such as The rated power of the engineand the corresponding speed,etc.Analogy must reference the same type of engine. Although the accuracy of this method is high, the practical application is limited[2].Engine characteristics estimation method in actual should be required to calculate the parameters relatively smaller, no complex calculations in the algorithm and can better describe the dynamic characteristics of the engine. The mean value model is employed to evaluate the gasoline engine character in this paper using MATLAB/Simulink software.

\section{MEAN VALUE MODEL}

Typical engine average model is proposed by Elbert Hendricks in 1989. Engine parameters described in model is the average value of this parameter in a certain period of time. This method not only applies to the steady state, but also applicable for transient. This model can accurately describe the engine air-fuel ratio. It has been widely used in the development of electronic engine control system[3]. The model is consists of the intake system sub-model, the dynamic fuel sub-model and power output sub-model. Algebraic equations and simple differential equations are used in each sub-model,so the model expression is simple and easy to understand.

\section{Intake system sub-model}

The intake system sub-model is core of the mean value model of the engine, and directly determine the overall mean value accuracy of the mode and intake air simulation accuracy created by the engine intake pressure. [4].

The air flow model on throttle. Air mass flow through the throttle is equivalent tothe product on the throttle $\boldsymbol{\beta}_{1}(\alpha)$ and pressure ratio $\boldsymbol{\beta}_{2}\left(\boldsymbol{P}_{\boldsymbol{r}}\right)$, so the original complex flow of variable cross-section can be simplify the flow of compressible gas in a one-dimensional steady-state contraction nozzle[5]. So the throttle at the air flow can be expressed as [6]:

$$
\dot{m}_{a t s}=\dot{m}_{a t 1} \beta_{1}(\alpha) \beta_{2}\left(P_{m}\right)+\dot{m}_{a t 0}
$$

Where:

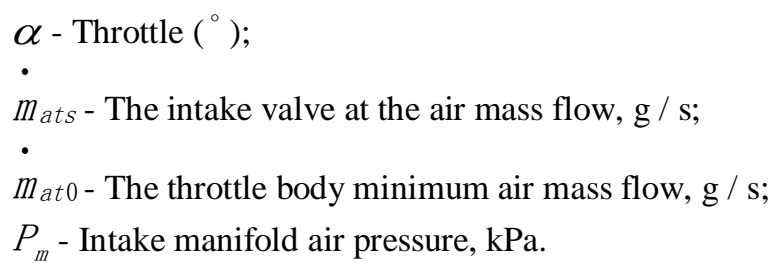




\section{The air flow model at the valve.}

The quality of the air entering the cylinder directly determines the amount of fuel injection within a cylinder, i.e. determines the injection pulse width of the engine ECU, and thus determine the output characteristics of the engine. The air flow rate at the intake valve is associated with engine speed, intake manifold pressure and temperature, the coefficient of charge etc,. The intake flowcan be used on speed - density equation [7].

$$
\dot{m}_{a p}=\frac{n}{120} V_{d} \rho \eta_{v o l}=\frac{n V_{d}}{120 R T_{m}} \eta_{v o l} P_{m}
$$

Where:

$n$ - Engine speed, $\mathrm{r} / \mathrm{min}$

$V_{d}$ - Engine displacement, $\mathrm{m}^{3}$

$\eta_{v o l}$ - Coefficient of charge, $\%$;

\section{The intake manifold air pressure model.}

In the intake manifold pressure model, the space from the throttle to the intake valve is as a whole.According to the ideal gas equation of state in this space, the following equation can be achieved:

$$
\dot{P}_{m}=\frac{R T_{m}}{V_{m}} \dot{m}_{a}
$$

In the engine intake system, air flows from the throttle to the intake manifold, and eventually flows into the cylinder through the intake valve. According to the mass conservation equation, air mass flow equationwithin the space of the intake manifold can be achieved as follows.

\section{Dynamic fuel sub-model}

$$
\dot{m}_{a}=\dot{m}_{a t}-\dot{m}_{a p}
$$

The initial fuel evaporation model is proposed by Rasmussen, hereafterimproved by the Aquino, and ultimately collate and analyze more comprehensive film evaporation modelby Elbert Hendricks [8].In order to make the cylinder into the fuel flow rate $\dot{m}_{f}$ is equal to a desired target injection quantity $\dot{m}_{f u}$, and then calculate the fuel injector fuel injection quantity. Let $\dot{m}_{f}$ equal to $\dot{m}_{f u}$, the oil film compensation model isgiven in equation (5).

$$
\left\{\begin{array}{l}
\dot{m}_{f i}=\frac{\dot{m}_{f u}-\dot{m}_{f f}}{1-X} \\
\ddot{m}_{f f}=\frac{1}{\tau_{f}}\left(-\dot{m_{f f}}+X \dot{m}_{f i}\right)
\end{array}\right.
$$

\section{Power output sub-model}

Power output model describes the energy flow in the fuel combustion in the engine. According to the law of conservation of energy, dynamic model of the gasoline is proposed by Hendricks in 1990, and still in use today. its expression is

$$
\frac{d}{d t}\left(\frac{1}{2} I \times n^{2}\right)=\operatorname{In} \dot{n}=-\left(P_{f}+P_{p}+P_{b}\right)+H_{\mu} \eta_{i} \dot{m}_{f}\left(t-\tau_{d}\right)
$$

Where:

$I$ - Inertia of crankshaft and load moment, $\mathrm{kg} / \mathrm{M}^{2}$;

$P_{f}+P_{p}$ - Friction and pumping losses power, $\mathrm{W}$;

$P_{b}$ - Load power consumption, $\mathrm{W}$;

$H_{\mu}$ - The fuel of low calorific value of $\mathrm{J} / \mathrm{kg}$;

$\eta_{i}$ - Indicated efficiency;

$\tau_{d}$ - Injection to speed response time delay, s, $\tau_{d}=60 / n$ 
III. THE OVERALL OFMEAN VALUE MODEL

Model of top-level, including three sub-models: the Air model represents the intake system model, Fuel modelrepresentative of the dynamic fuel model and thePower modelon behalf of power output model. Intake pressure and intake air flow is obtained with the throttle and speed changes in model of Air model. The inlet pressure is used for model inputs of Fuel model and Power model, Whichprovides the conditions for calculating the fuel injection quantity and the final power output. The final output characteristics of the engine under various conditions can be achieved in the entire model by throttle $(\alpha)$, engine speed $(\mathbf{n})$, the model shown in Figure 2.

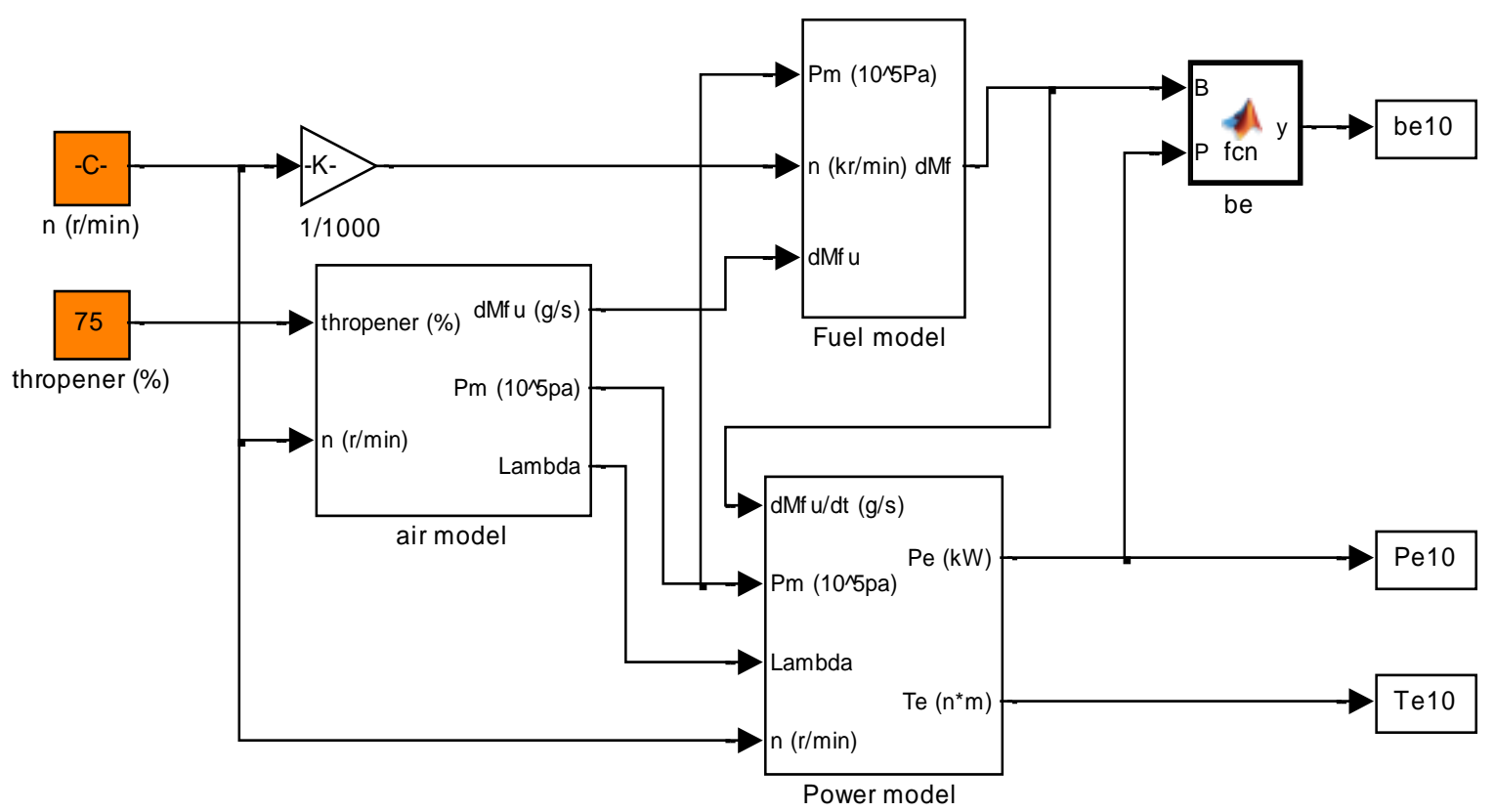

Figure 2Overall block diagram of mean value model

\section{SIMULATION RESULTS AND COMPARATIVE ANALYSIS OF TEST RESULTS}

Based on simulation platform of MATLAB/Simulink module, engine power output characteristics, the external characteristic of speed and torque-speed characteristics of the engine of Hyundai 1.8L four-cylinderare calculated. Compared with the bench test results, torque error of engine power output is small, theaccuracy model is higher, and it well predict power of the engine output Characteristic. The graph of the engine power, torque the outer characteristics is given in figure 3 , the model calculation of the proceeds of the engine maximum torque which calculated by model has same values with data given by the manufacturers within allowable range.
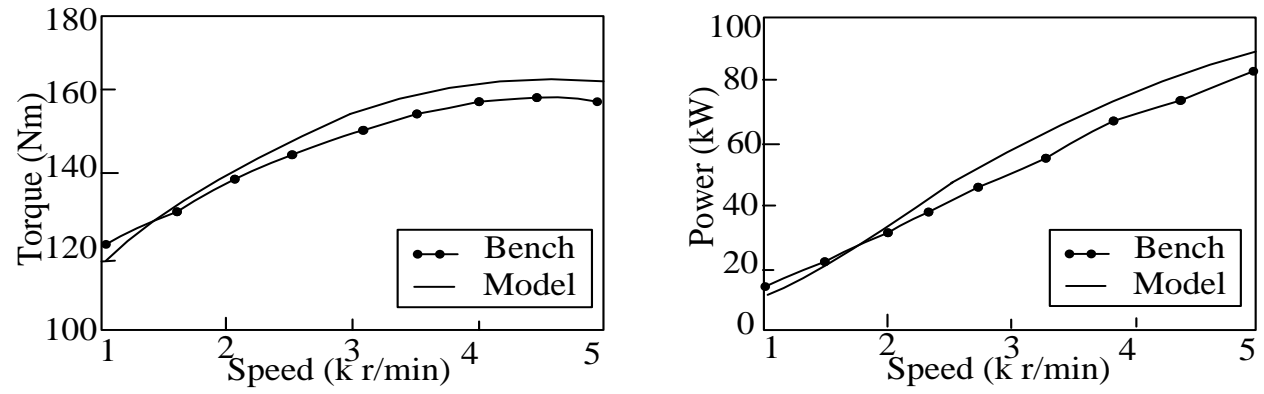

Figure 3Engines external characteristics compared with the bench test

\section{CONCLUSION}

In this paper, parameters of enginemean value model, such as the engine throttle opening, and volume of the intake manifold, intake air pressure and temperature, intake air flow, the amount of fuel injection is research,and the engine power output characteristics predictive model is establishusing MATLAB/Simulink module.The simulation results of the model is compared and analysis with bench test data. The results show that 
the model can be better described engine output power and torque, the curve trend of the curve obtained by simulation with the test proceeds is basically the same, the error is small, and this model can satisfy the basic requirements of the engine control technology simulation.

\section{ACKNOWLEDGEMENTS}

This work was financially supported by the National Natural Science Foundation (51175450), Hebei Province Natural Science Foundation (E2012203046).

\section{REFERENCES}

[1] MaozhaoXie. Internal combustion engine to calculate the combustion. Dalian: Dalian University of Technology Press, 2005:4-87, In Chinese.

[2] JingmingZhang, GuifanZhao, LibiaoJiang. Engine characteristics to calculate the model vehicle performance computing. Car engines, 2010, (3) 26-28,In Chinese.

[3] Alain Chevalier, Martin Muller, Elbert Hendricks. On the Validity of Mean Value Engine Models During Transient Operation. SAE TECHNICAL PAPER SERIES 2000-01-1261, 2000.3:1-24.

[4] FengWu, JinlongHu, BowenZou. Simulation and experimentalstudy ofEFI gasoline's manifold filling dynamics model. Chinese internal combustion engine engineering, 2006, (3)25-28, in Chinese.

[5] Rob Karmiggelt. Mean value modelling of a SI engine. Eindhoven University of Technology, 2008.10:4-9.

[6] Chao Chen, Shaoguang Wang, Xiaodun Kang. Electronic Fuel control system based on Matlab/Simulink simulation of the internal combustion engine works, 2009, (5):23-27, in Chinese.

[7] Elbert Hendricks, Spencer C, Sorenson. Mean Value Modeling of Spark Ignition Engines. SAE Technical Paper Series 900616, 1990.2:1-15.

[8] Munan Hong, OuyangMinggao. Estimated based on the average gasoline engine model line torque. Of Mechanical Engineering, 2009, (4):290-294, in Chinese. 